\title{
Clinical applications of dynamic functional musculoskeletal ultrasound
}

This article was published in the following Dove Press journal:

Reports in Medical Imaging

20 February 2014

Number of times this article has been viewed

Jonelle Petscavage-Thomas

Department of Radiology, Penn State Hershey Medical Center, Hershey, PA, USA
Correspondence: Jonelle PetscavageThomas

Department of Radiology - HG300], Penn State Hershey Medical Center, 500 University Drive, Hershey, PA, 17033 USA

Email jthomas5@hmc.psu.edu

\begin{abstract}
There is an increasing trend in medicine to utilize ultrasound for diagnosis of musculoskeletal pathology. Although magnetic resonance imaging provides excellent spatial resolution of musculoskeletal structures in multiple imaging planes and is generally the crosssectional modality of choice, it does not provide dynamic functional assessment of muscles, tendons, and ligaments. Dynamic maneuvers with ultrasound provide functional data and have been shown to be accurate for diagnosis. Ultrasound is also less expensive, portable, and more readily available. This article will review the common snapping, impingement, and friction syndromes imaged with dynamic ultrasound. It will also discuss future areas of research, including musculoskeletal sonoelastography.
\end{abstract}

Keywords: snapping, dynamic, ultrasound, functional, musculoskeletal

\section{Introduction}

Ultrasound image resolution has substantially improved over the past few decades, enabling increased clinical application. Unlike magnetic resonance (MR) and computed tomography (CT) imaging, which provide structural information, sonography allows acquisition of dynamic information. In dynamic ultrasound imaging, the patient performs a movement while the physician holds the ultrasound probe relative to an anatomic landmark. ${ }^{1}$ This has particularly useful applications for musculoskeletal (MSK) imaging, where several pathological conditions are elicited only through patient movement. Ultrasound also offers the benefits of increased accessibility, lower cost, and no use of ionizing radiation.

There has been significantly increased utilization of MSK ultrasound over the past decade, with a recent study showing a $316 \%$ increase in the number of studies performed between 2000 and 2009. ${ }^{2}$ Another study of recent MSK radiology fellows found that $69 \%$ are performing ultrasound in their practices. ${ }^{3}$ Thus, knowledge of the common dynamic ultrasound techniques and recent advances in this realm are important as this field grows. This article will review clinical applications of dynamic MSK ultrasound. The article will discuss the major uses of dynamic ultrasound for each joint.

\section{Dynamic dysfunction categories Snapping}

Snapping occurs from impingement of a structure against another anatomic or heterotopic structure. ${ }^{4}$ It is associated with abrupt movement and a clicking or snapping noise. 
Snapping syndromes may also be called clunking, locking, or triggering. Patients may require surgical intervention in cases of severe pain or dysfunction. Examples include trigger finger and snapping hip syndromes. Ultrasound is particularly useful in snapping syndromes.

\section{Friction}

Friction syndromes occur from a smooth impingement, resulting in pain, but without the audible snaps or clicks. ${ }^{4}$ Examples include intersection syndrome of the wrist and iliotibial (IT) band syndrome at the knee. Although ultrasound may demonstrate static findings similar to MR imaging (MRI), dynamic imaging is proven and applicable in certain conditions, but not well researched in others.

\section{Shoulder}

\section{Subacromial impingement}

A common indication for dynamic imaging at the shoulder joint is in the evaluation of subacromial impingement. Neer first described subacromial impingement in 1972. ${ }^{5}$ Impingement occurs when the greater tuberosity or soft tissues encroach on the coracoacromial arch in abduction, flexion, and internal rotation. ${ }^{5}$ It is a common cause of shoulder pain, and is seen in repeated overhead activities, such as weightlifting, swimming, and tennis. ${ }^{6}$ Dynamic imaging is helpful in confirming clinical suspicion. Ultrasound is $79 \%$ sensitive and $88 \%$ specific for impingement of the supraspinatus tendon. ${ }^{7}$

In dynamic ultrasound, a linear high-frequency transducer $(7-12 \mathrm{MHz})$ is placed at the anterolateral edge of the acromion in the oblique coronal plane. The patient abducts the arm anterolaterally while held in internal rotation (thumb down). ${ }^{8}$ In mild clinical impingement, ultrasound is normal. In patients with moderate subacromial impingement, images will demonstrate subacromial subdeltoid bursal fluid located laterally to the acromion (Figure 1). ${ }^{8,9}$ Additionally, the supraspinatus tendon will fail to pass over the greater tuberosity in active elevation of the arm halfway between flexion and abduction. Ultrasound findings in severe impingement include superior migration of the humeral head and bunching or bulging of the supraspinatus tendon laterally. ${ }^{8}$

\section{Adhesive capsulitis}

Another indication for dynamic MSK ultrasound is in the diagnosis of adhesive capsulitis. Adhesive capsulitis, also known as frozen shoulder, is characterized by symptomatically progressive pain and contracture. ${ }^{10}$ The movements of external rotation and abduction, such as when combing

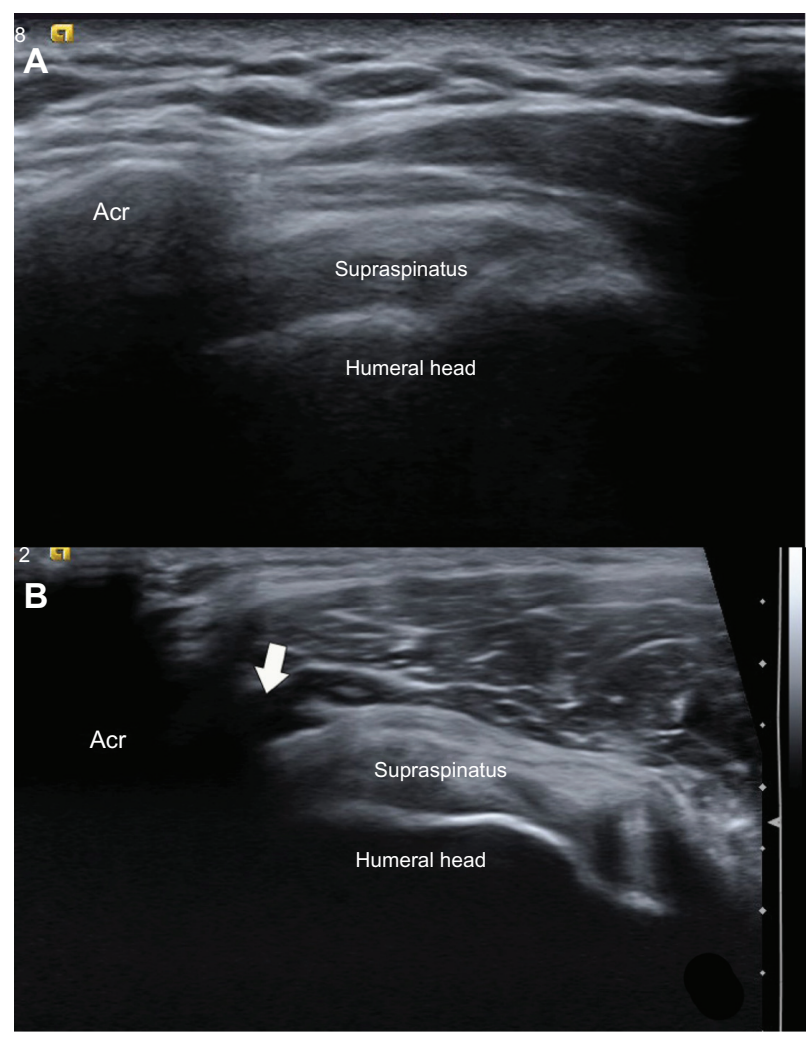

Figure I (A and B) Subacromial impingement. (A) Coronal sonography view of the right shoulder during active elevation of arm halfway between flexion and abduction and hand in pronation shows the normal gliding of the tendon underneath the acromion, without pooling of fluid in the subacromial-subdeltoid bursa. (B) Coronal sonography view of the right shoulder during same dynamic maneuver in a symptomatic patient shows abnormal pooling of fluid in the lateral aspect of the subacromial-subdeltoid bursa (arrow) and mild impingement of supraspinatus tendon.

Abbreviation: Acr, acromion.

the hair, or extension and internal rotation, such as when reaching for a bra strap, may elicit the pain. ${ }^{10}$ The condition affects middle-aged individuals, slightly more often in women. Although there are typical MR arthrography findings, ultrasound has been used to diagnosis adhesive capsulitis.

Ryu et al reported that painful gliding of the supraspinatus in the subacromial space on dynamic ultrasound indicated decreased joint capacity or joint stiffness. ${ }^{11}$ A study by Kim et al further evaluated this theory by comparing dynamic ultrasound images with the gold standard of magnetic resonance arthrography (MRA). ${ }^{12}$ The authors demonstrated a strong linear relationship between painful gliding of the supraspinatus in the subacromial space and MRA features of coracohumeral ligament thickening $>4 \mathrm{~mm}$, thick joint capsule in the rotator interval, and thickening of the synovium and capsule at the level of the axillary recess. ${ }^{12}$ In dynamic imaging, the patient is placed in the decubitus position with the arm in pronation. A linear high-frequency transducer is positioned between the greater tuberosity and acromion, and 
the arm is raised in abduction, looking for painful gliding of the tendon.

\section{Biceps tendon subluxation/dislocation}

Dynamic ultrasound is also beneficial in evaluating transient subluxation and dislocation of the long head biceps (LHB) tendon. Normally, the tendon is localized to the intertubercular groove of the humeral head, stabilized by the transverse ligament and supraspinatus and subscapularis tendons. The LHB tendon may subluxate or dislocate medially if there is a subscapularis tendon tear, the coracohumeral or glenohumeral ligament is torn, or the bicipital groove is congenitally shallow. This condition often requires surgical intervention, whereas biceps tendon tears without subluxation can be treated conservatively. ${ }^{13} \mathrm{~A}$ study by Armstrong et al compared ultrasound findings to surgical findings and found sonography reliable in detecting subluxation and dislocation. ${ }^{14}$ Dynamic ultrasound has also been shown to be more accurate than MRA for LHB tendon subluxation or dislocation and to detect $86 \%$ of LHB tendon subluxations. ${ }^{15,16}$

In dynamic ultrasound, the transducer is positioned transversely over the bicipital groove. The patient moves the shoulder to complete external rotation and a slight amount of abduction. ${ }^{8}$ In neutral, the LHB tendon is visualized as a hyperechoic and fibrillar tendon of uniform thickness. If abnormal, the LHB tendon is seen perching onto the lesser tuberosity medially in subluxation (Figure 2 ). ${ }^{8}$ In dislocation, the tendon moves medially into the joint or substance of the supraspinatus tendon.

\section{Snapping scapula}

Snapping scapula is an uncommon entity. Patients complain of an audible snapping with painful crepitus. ${ }^{8,17}$ The underlying etiology can be soft tissue or skeletal, and is often seen with repetitive overhead motions of the arm, particularly in the young active patient population. ${ }^{17}$ Although radiographs may show an osseous cause of snapping scapula, such as an osteochondroma, the lesions may not be readily visible. CT interpretations have not been shown to correlate well with clinical findings. ${ }^{18}$ With dynamic ultrasound, the sonographer will visualize movement of the scapula against the rib cage and possibly an underlying soft-tissue cause, such as bursitis or muscle atrophy. This can also aid in ultrasoundguided bursal injection. ${ }^{17}$

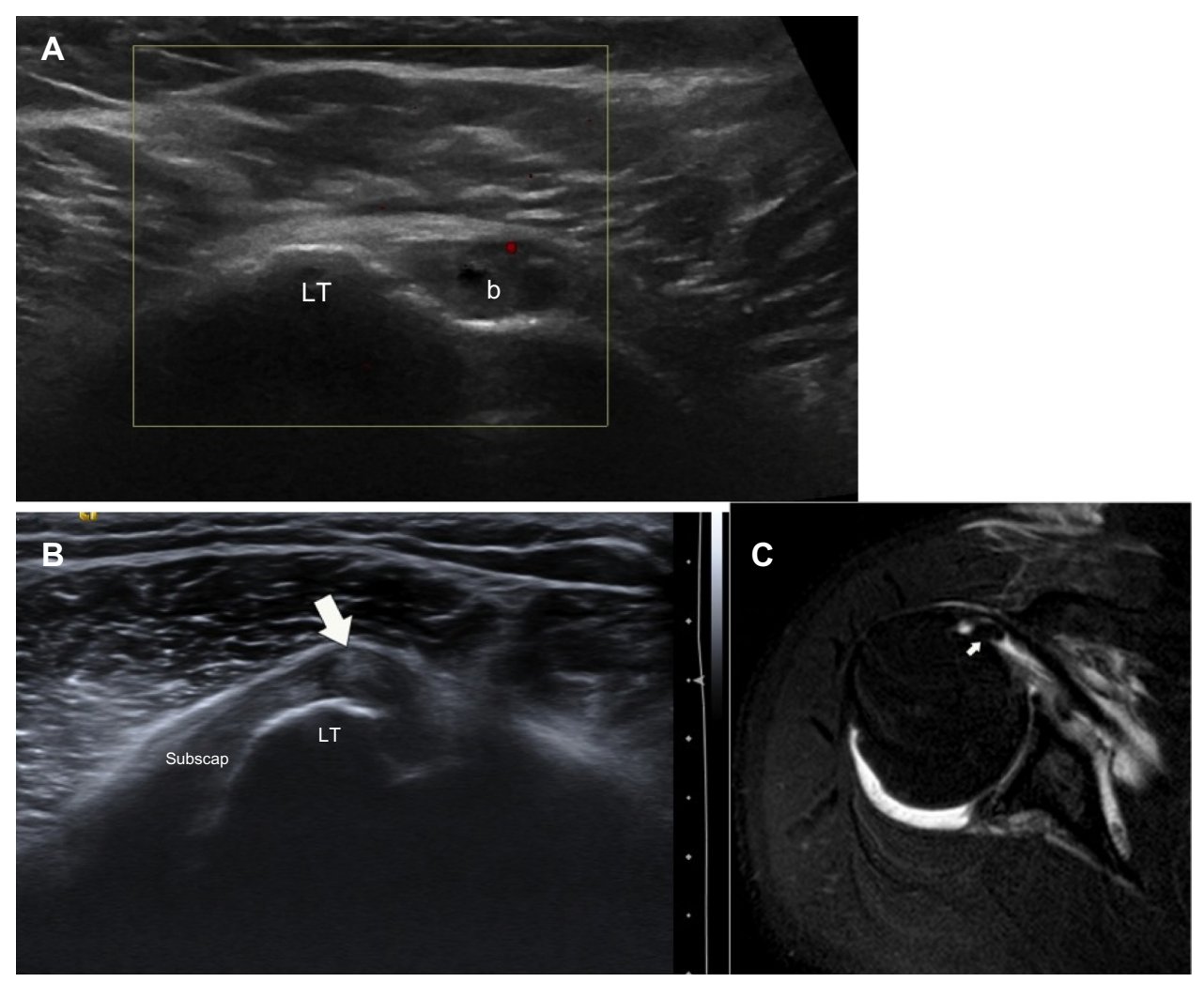

Figure 2 (A-C) Long head biceps subluxation. (A) Transverse ultrasound of the left shoulder in external rotation shows normal position of the long head biceps tendon (b) in the bicipital groove. (B) Transverse ultrasound of the left shoulder in external rotation in a different patient shows the abnormal perching of the long head biceps tendon (arrow) on the lesser tuberosity (LT) out of the bicipital groove, consistent with subluxation. (C) Magnetic resonance arthrogram axial $\mathrm{T}_{2}$ fat-suppressed image confirms the subluxation of the biceps tendon in this patient. 


\section{Elbow}

\section{Ulnar collateral ligament injury}

The ulnar collateral ligament (UCL), also known as the medial collateral ligament, is the primary stabilizer of the elbow against valgus forces. ${ }^{19}$ The anterior bundle of the UCL is the strongest and originates on the medial epicondyle of the humerus and inserts onto the sublime tubercle. It is crucial in the overhead-throwing athlete for late cocking and early acceleration phases. ${ }^{20}$ Unfortunately, injury to the UCL has become more prevalent among high school, collegiate, and professional baseball players. ${ }^{19}$

Dynamic ultrasound is particularly useful to demonstrate laxity of the UCL and joint instability associated with partialand full-thickness tears. Application of valgus stress on the elbow demonstrates abnormal widening of the medial joint space with UCL injury. ${ }^{21,22}$ For ultrasound technique, the shoulder is in $90^{\circ}$ of flexion, and the elbow is placed in $25^{\circ}$ of flexion with the transducer held longitudinally. An assistant presses on the lateral elbow and pulls laterally on the distal forearm to mimic valgus stress (Figure 3). ${ }^{21}$

Normally, the UCL is a moderately echogenic band of tissue extending from the distal end of the medial edge of the coronoid. Ultrasound will demonstrate focal hypoechoic areas in partial tears and complete disruption of the tendon in full-thickness tears. Ultrasound diagnosis of

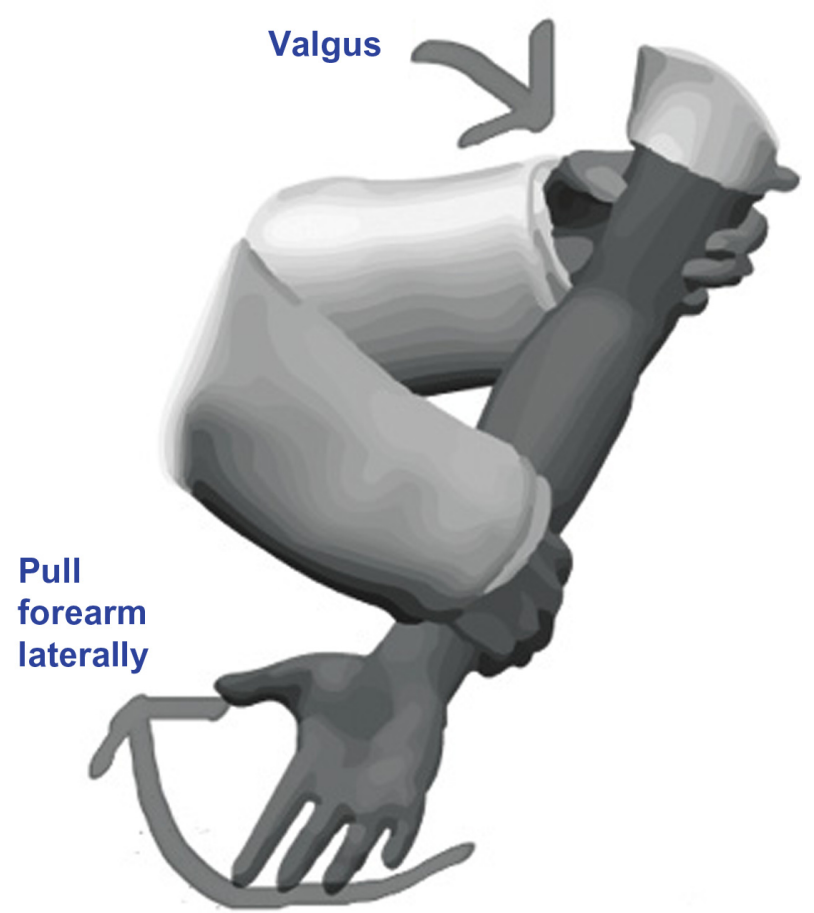

Figure 3 Author illustration of dynamic technique for assessing for laxity of the ulnar collateral ligament of the elbow. Valgus stress is placed on the elbow while the forearm is pulled laterally. instability is seen with medial joint laxity of $>1 \mathrm{~mm}$ under valgus stress. ${ }^{21}$

\section{Snapping triceps/ulnar nerve subluxation}

Snapping triceps is an uncommon condition in which the triceps tendon dislocates over the medial epicondyle of the elbow with flexion or extension. ${ }^{23}$ Patients experience focal tenderness and snapping along the medial side of the elbow. A smaller than normal size interval between the medial edge of the triceps and the medial humeral epicondyle predisposes to snapping, as does an abnormal myotendinous portion of the triceps in the ulnar groove. ${ }^{23}$

Coexistent ulnar nerve dislocation often occurs. ${ }^{24}$ This can result in pain and tingling numbness in the ulnar nerve distribution. ${ }^{25}$ The ulnar nerve at the elbow is located in the cubital tunnel, which is formed by the cubital retinaculum and medial epicondyle. The nerve runs between the medial edge of the triceps and the medial humeral epicondyle. A shallow groove or insufficiencies of the retinaculum are potential causes of a dislocating ulnar nerve..$^{25}$ The condition is also seen in bodybuilders, abnormal triceps tendons, and posttrauma. ${ }^{26}$ Dislocation occurs if the nerve slides out of the cubital tunnel and over the medial epicondyle during elbow flexion. Ulnar nerve subluxation/dislocation can occur independently of a snapping distal triceps tendon.

Both of these pathologies can be seen with dynamic ultrasound as the probe is held in the transverse plane between the olecranon and medial epicondyle, and the patient flexes and extends the arm..$^{22}$ In flexion, there is abnormal anterior and medial translation of the ulnar nerve over the medial epicondyle (Figure 4A and B). ${ }^{22}$ The ulnar nerve also flattens during flexion in patients with subluxation. ${ }^{26}$ This may be accompanied by abnormal anterior translation of the medial head of the triceps muscle (Figure $4 \mathrm{C}$ and D). Longitudinal dynamic imaging during flexion and extension is useful to differentiate partial from nonretracted complete distal triceps tendon tears.

\section{Wrist and hand Extensor carpi ulnaris subluxation}

The extensor carpi ulnaris (ECU) tendon is the sixth dorsal wrist compartment. The tendon has an angular path across the ulnar head to its insertion onto the base of the fifth metacarpal, increased with ulnar deviation. ${ }^{27}$ The tendon is stabilized in the ulna groove by its own subsheath. Although there is some degree of normal subluxation of the tendon out of the groove, larger degrees are associated with ulnar-sided wrist pain..$^{27,28}$ This entity is often seen in golfers, racquetball, 


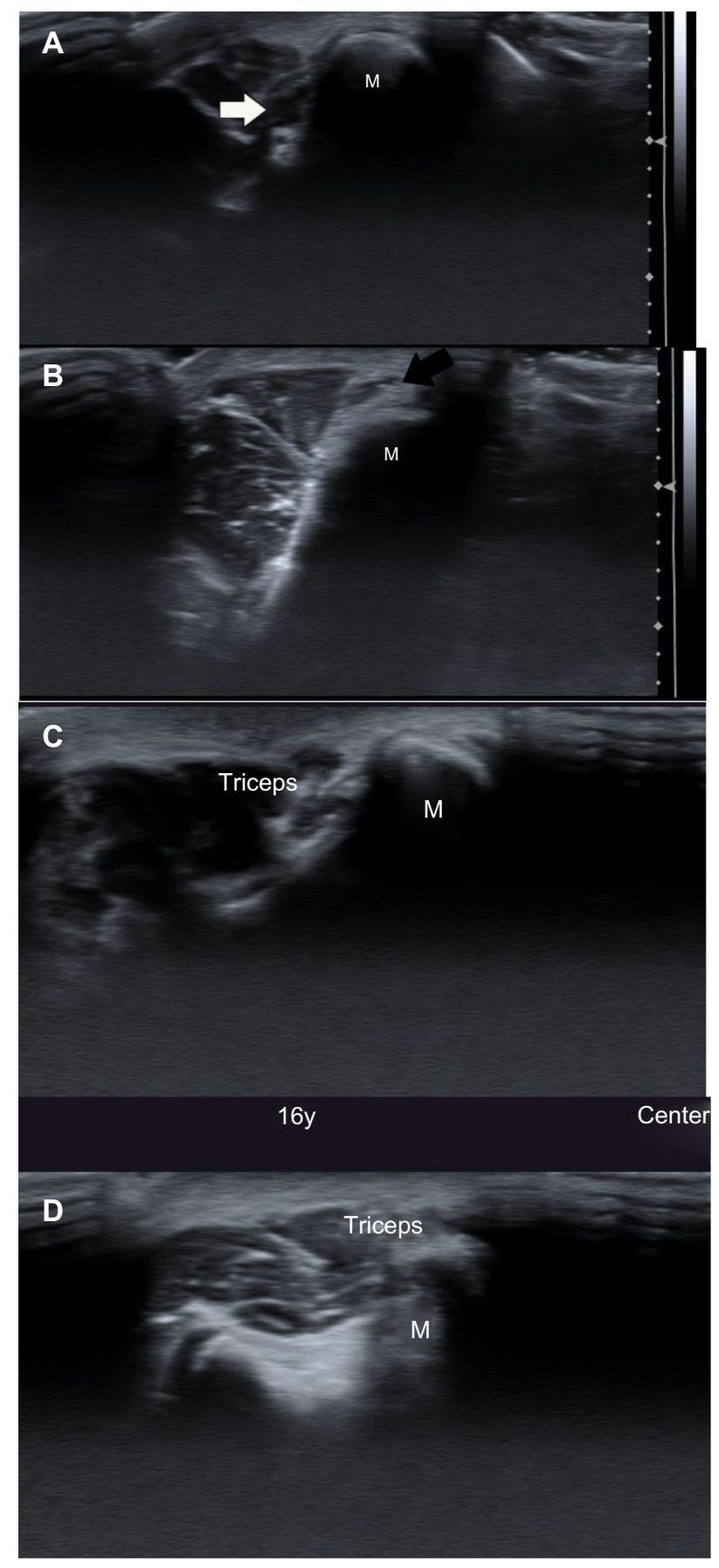

Figure 4 (A-D) Ulnar nerve subluxation. (A) Transverse ultrasound of the cubital tunnel in extension shows normal position of the ulnar nerve (white arrow). (B) Transverse dynamic ultrasound image in flexion shows anteromedial subluxation of the ulnar nerve (black arrow) over the medial epicondyle. (C) Transverse ultrasound of the cubital tunnel in extension in a different patient shows normal position of the triceps. (D) With flexion, the triceps moves over the medial epicondyle (M) with audible snap on dynamic ultrasound.

or tennis players. Patients report an audible snapping or clicking sound in forearm rotation.

Since the subluxation is based on motion, dynamic ultrasound imaging has been suggested as the best modality for reproducing clinical symptoms. In a study of 21 patients, ultrasound correctly identified subluxation in all 21 when compared to surgical results. ${ }^{27}$ On dynamic ultrasound, a highfrequency linear transducer is placed over the distal ulna in the transverse plane. With supination, an unstable tendon moves abruptly over the ulnar aspect of the groove and subluxates volarly, reducing back to normal in pronation (Figure 5). ${ }^{29}$ The subluxation increases with supination, flexion, and ulnar deviation. ${ }^{30}$ Normal subjects will never have subluxation beyond the apex of the ulnar border of the groove. ${ }^{30}$

\section{Trigger finger}

Trigger finger clinically presents as a locking and or clicking of the flexor tendons of the hand. ${ }^{8}$ Patients experience discomfort in the palm that increases to painful popping or snapping as the patient flexes and extends the digit. Some patients experience a locking of the finger in flexion..$^{31}$ The condition is seen most commonly in middle-aged women and those with rheumatoid arthritis and diabetes mellitus. ${ }^{32}$ The pathophysiology is not completely understood. ${ }^{33}$

Trigger finger is a stenosing tenosynovitis that has specific ultrasound findings. Normally, the flexor tendons have a normal fibrillar pattern, and annular pulleys are hyperechoic structures about the volar aspect of the tendon sheath. ${ }^{33}$

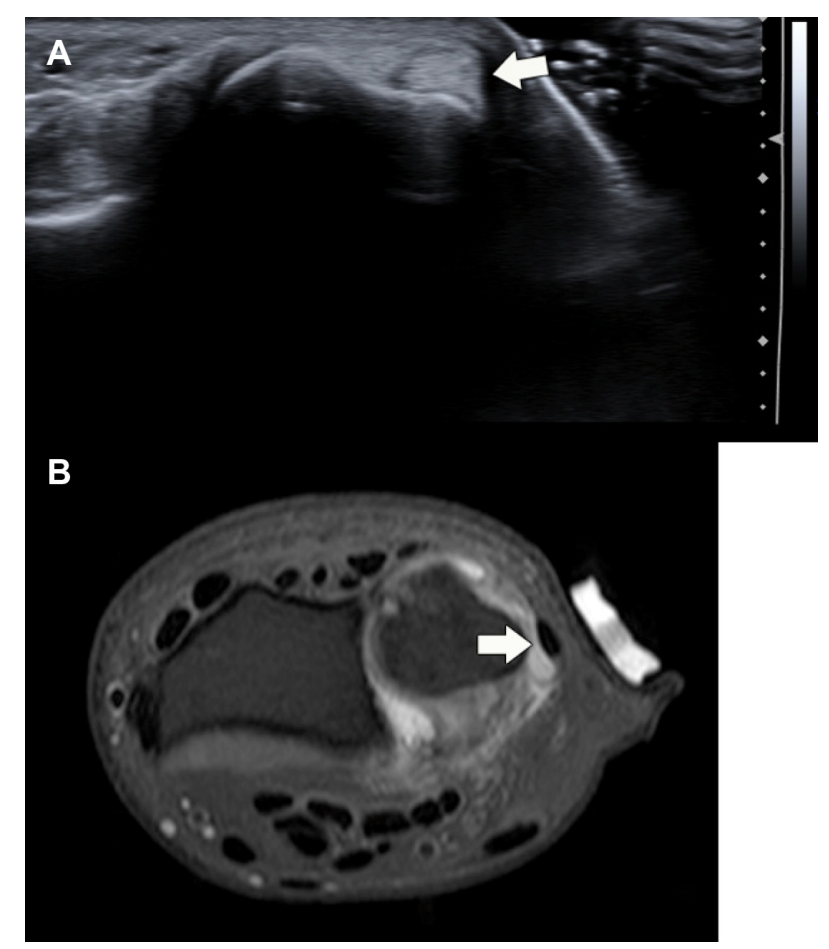

Figure 5 (A and B) Extensor carpi ulnaris subluxation. (A) Transverse ultrasound image of the distal ulna and extensor carpi ulnaris shows volar subluxation of the tendon halfway between pronation and supination. (B) Axial proton-density fatsuppressed magnetic resonance image of the same patient 3 months later shows the tendon is now dislocated in neutral position. 
In trigger finger, static ultrasound images may demonstrate thickening of the synovial sheath, abnormal hypoechoic sheath echotexture, tendon thickening, or a hypoechoic thickened A1 pulley at the level of the metacarpophalangeal joint on the volar aspect of the tendon sheath. ${ }^{34}$

With the transducer oriented longitudinally to the tendon, dynamic imaging in finger flexion and extension demonstrates impingement of the thickened tendon and sheath with lack of a smooth gliding motion during patient movement (Figure 6A and B). There is a blurring margin of the tendon under the A1 pulley with motion. ${ }^{35}$

\section{Boxer's knuckle}

Extensor tendon abnormalities can also benefit from dynamic imaging. Boxer's knuckle involves traumatic disruption of the extensor tendon hood from sagittal band injury. ${ }^{36}$ Sagittal bands are transversely oriented ligaments stabilizing the extensor tendons of the finger. Patients experience pain, decreased joint extension, and swelling. Palpable subluxation of the tendon in flexion may be difficult due to swelling. ${ }^{37}$ Subluxation can also occur in nontraumatic situations, such as patients with inflammatory arthritis or congenital deficiencies. ${ }^{37}$

With the ultrasound transducer held transverse at the level of the metacarpophalangeal joint, the patient flexes the joint. In Boxer's knuckle, the extensor tendon will subluxate ulnarly or radially relative to the metacarpal head (Figure 6C and D). ${ }^{8}$ Tenosynovitis and tendon split tears, seen as hypoechoic foci, may also be present.
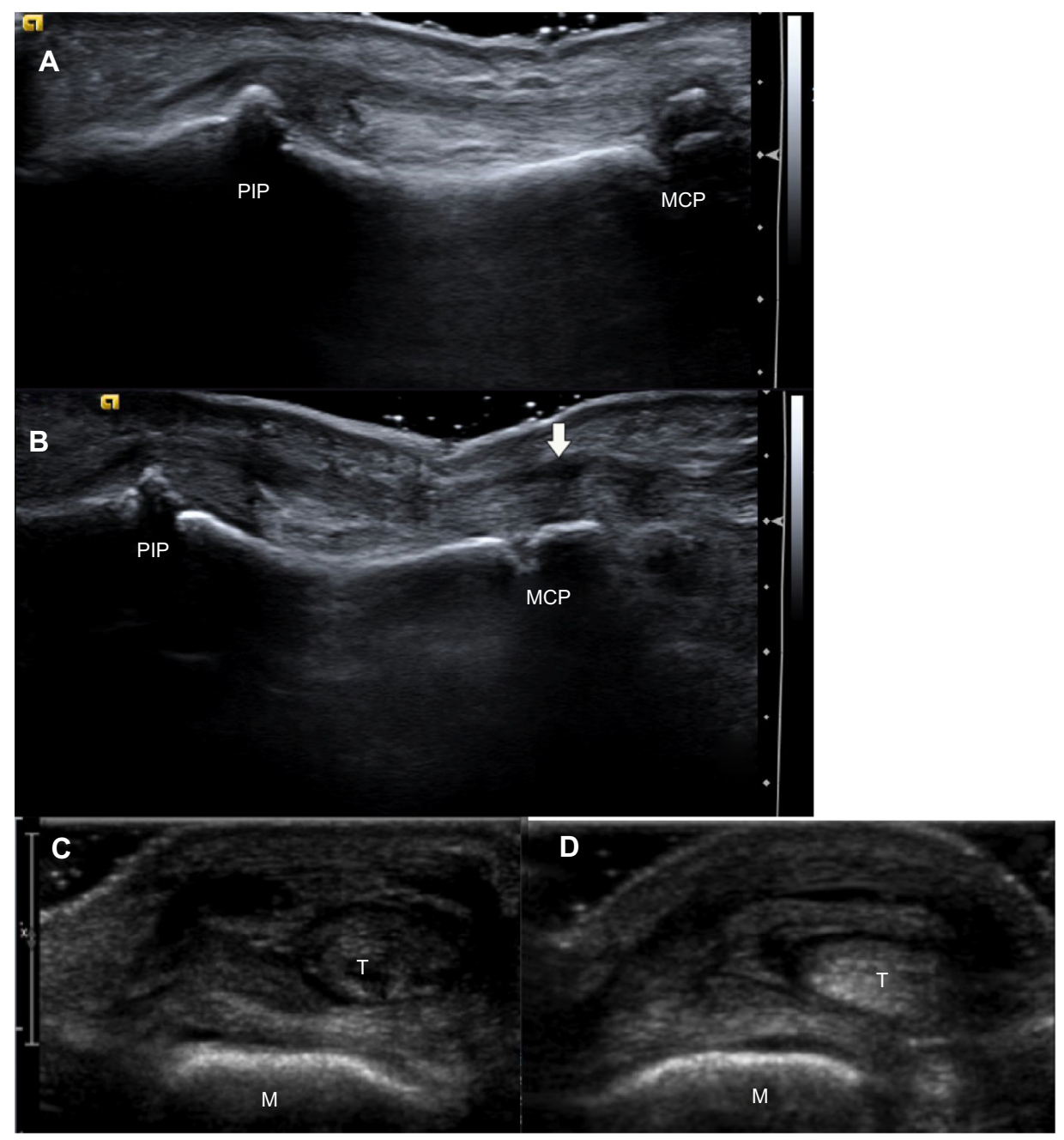

Figure 6 (A-D) Dynamic finger imaging. (A) Pre- and (B) postflexion longitudinal ultrasound images of the third flexor tendon at the level of the metacarpophalangeal (MCP) joint demonstrates lack of smooth gliding of the tendon, with relatively unchanged position. Findings are consistent with trigger finger. There is also decreased echotexture of the Al pulley (arrow) and blurring of the tendon under the pulley, with an additional finding of trigger finger. (C) Transverse ultrasound image shows radial deviation of fourth extensor tendon (T) at the level of the metacarpal head (M) in neutral. (D) Clenching of the patient's fist accentuates the radial deviation of the extensor tendon. Findings are consistent with sagittal band rupture of the extensor tendon, known as Boxer's knuckle.

Abbreviation: PIP, proximal interphlalangeal. 


\section{Dupuytren's contracture}

Palmar fibromatosis is the most common superficial fibromatosis, typically affecting men over age 65 years. ${ }^{38}$ Palmar fibromatosis etiology is multifactorial, including traumatic, microvascular, immunological, and genetic factors. Clinically, patients complain of painless subcutaneous nodules along the volar aspect of the finger. Over time, these may grow deeper and attach to and cause traction on underlying flexor tendons. On static ultrasonography, palmar fibromatosis nodules are hypoechoic, hypervascular, and superficial to the flexor tendons. ${ }^{38}$ Dynamic ultrasound allows assessment of the extent of flexor tendon contracture and confirms adherence of the nodules to the tendons.

\section{Hip}

There are both extra-articular and intra-articular causes of the clinical sensation of a snapping sound during flexion of the hip joint or while walking. ${ }^{4}$ This is often seen in young athletic adults and dancers. The snap may or may not be accompanied by pain. Intra-articular causes include labral tears, intra-articular loose bodies, osteochondral fracture, and transient subluxation of the femoral head. These are not assessed routinely under dynamic ultrasound..$^{4,8}$ Extra-articular causes include anterior and lateral/posterior snapping hip syndromes.

Anterior or internal snapping hip is seen as the iliopsoas tendon moves over the superior pubic ramus during activities that have movements of abduction, flexion, and external rotation of the hip. Initially, the pathophysiology described impingement between the psoas tendon and the iliopectineal eminence. ${ }^{39}$ More recent studies have shown alternatively a rotational motion of the tendon against the pubic ramus, not involving the iliopectineal eminence. ${ }^{40}$

Under sonography, the probe is placed transversely over the lesser trochanter. The patient starts in the frog-leg position of flexion, abduction, and external rotation, and then returns the hip from flexion back to full extension. A snap is heard halfway through motion. The images will show abrupt mediolateral or rotary motion of the iliopsoas tendon. The medial fibers of the iliacus can be seen caught between the superior pubic ramus and psoas tendon in frog-leg position and freed as it abruptly snaps against the bone in return to full extension. ${ }^{41}$ An underlying synovial cyst may be present.

Lateral cause of snapping hip includes abnormal movement of a thickened posterior border of the IT band or anterior border of the gluteus maximus over the greater trochanter. ${ }^{4}$ This occurs over the greater trochanter during the return of hip from flexion to full extension. The condition is associated with coxa vara, limb-length discrepancy, hardware impingement, and a thick junction between the tract and gluteus maximus muscle. With the transducer in the axial plane positioned over the great trochanter, the patient lies in lateral decubitus. With hip flexion, snapping is reproduced with abnormal jerky anterior motion of the tensor fascia lata. ${ }^{8}$

Posterior snapping is a rare entity that occurs as the long head of the biceps femoris impinges against the ischial tuberosity. ${ }^{4}$ Ischiofemoral impingement occurs with quadratus femoris tendon impingement between the lesser trochanter and ischial tuberosity. It results in buttock pain with intermittent snapping. Most research has described ultrasound findings of $<2 \mathrm{~mm}$ ischiofemoral space and asymmetric atrophy of the quadratus femoris muscle. ${ }^{4}$ Although not yet described by dynamic ultrasound, there is potential, given easy visualization of the structures with high-frequency linear transducers. Theoretically, with the hip in adduction, external rotation, and motion from flexion to extension, narrowing of the ischiofemoral space and reproduction of a snap could occur.

\section{Knee and calf Snapping knee}

Several entities are described as a snapping knee, and can be categorized into intra-articular and extra-articular causes.

\section{Medial extra-articular snapping}

Extra-articular snapping can be further divided into medial and lateral entities, and are well demonstrated with dynamic ultrasound imaging. Medially, a painful snap occurs as the gracilis or semitendinosus tendons pass over the medial tibial condyle. ${ }^{42}$ This is also sometimes called snapping pes anserine. The snap occurs at $15^{\circ}$ of extension when the knee is returning from a flexed position. It is seen in knee recurvatum, volleyball players, women wearing high heels, and posttraumatic hypertrophy of the pes anserinus and exostosis of underlying medial tibia. Dynamic ultrasound involves transverse orientation of the probe again the medial proximal tibia, with the patient returning from flexion to extension. ${ }^{43}$ An abnormal course of the tendons along the bone with an audible snap is reproduced.

\section{Lateral extra-articular snapping}

Laterally, a snap can occur as the biceps femoris flips over the fibular head during extension of the knee to $90^{\circ} .{ }^{44}$ This syndrome is seen in young men engaged in running, bicycling, and soccer. ${ }^{43}$ This may occur due to an anomalous insertion of the tendon onto the fibular head or anterolateral 
aspect of the proximal tibia. ${ }^{44}$ With the probe in the axial plane, the biceps is visualized at the level of the fibular head while the patient flexes and extends the knee by $90^{\circ} .{ }^{44}$ The jerky movement of the tendon can be visualized and a concurrent snap heard.

Laterally, the popliteus tendon can cause a snap as it moves from $0^{\circ}$ to $90^{\circ}$ of flexion due to abrupt extrusion of the tendon along the edge of the popliteal groove/lateral femoral condyle groove..$^{45}$ This condition is seen in the same population as biceps femoris snapping syndrome, but underlying causes are different and include osteophytes or total knee arthroplasty components. The transducer should be placed in the middle of a line joining the lateral epicondyle to the lateral joint line. In the axial plane, a jerky motion may be seen between flexion and extension of the knee. ${ }^{43}$

\section{IT-band friction syndrome}

IT-band friction against the lateral femoral condyle occurs at about $30^{\circ}$ of knee flexion. ${ }^{4,8}$ It is commonly seen in runners. On ultrasound, a nonjerky motion of the tendon can be elicited with knee flexion with the transducer held transversely. ${ }^{46}$ Thickening of the tract and adjacent soft-tissue fluid may be seen.

\section{Intra-articular snapping}

\section{Meniscus}

Causes of intra-articular snapping include foreign bodies, plicae, meniscal tears, and tumors. Although most are best imaged with MRI, the snapping meniscus can be seen with dynamic ultrasound.

Meniscal instability associated with meniscal tears can result in pain, locking of the knee, and isolated snapping. Meniscal instability is dynamically viewed with the probe located longitudinally over the joint line, looking for extrusion of the meniscus. The degree of medial meniscal subluxation has been shown to correlate with the radiographic degree of narrowing in the medial tibiofemoral joint space. ${ }^{47,48}$ For dynamic ultrasound, a linear transducer is positioned along the long axis of the knee at the level of the medial collateral ligament. The patient moves the knee into full extension in the supine position, and measurements can be obtained. Then, repeat imaging can be done with weight-bearing. A quantitative means of measuring the degree of excursion involves measuring the displacement of the medial collateral ligament (MCL), which is easier to see in its entirety than the meniscus and which is adherent to the peripheral edge of the meniscal body. ${ }^{49}$ Thus, with meniscal subluxation, the MCL will also move concordantly towards the parameniscal recess of the joint. ${ }^{8}$

\section{Snapping plica}

Plicae are remnant of intra-articular membranes of early fetal life. Suprapatellar, medial, and infrapatellar plica are the three types, and can occasionally become painful and produce a snap in patients with trauma or repetitive strenuous exercise. Specifically, thickening and loss of elasticity of the suprapatellar plica can cause impingement as it moves over the femoral condyle. ${ }^{4}$ On dynamic ultrasound, the suprapatellar plica will be seen in the transverse plane with abnormal rotational movement when the patient squats or transitions between sitting and standing. ${ }^{4}$

\section{Patellar clunk syndrome}

Another anterior source of knee pain is the "patellar clunk" syndrome. This is the most common etiology of a snapping phenomenon in a patient with total knee replacement. ${ }^{8}$ The condition is more prevalent in posterior stabilized total knee replacement, and occurs within a few months to years after surgery. Patients complain of painful catches or audible snaps above the patella during extension from $45^{\circ}$ flexion. This results from an abnormal suprapatellar fibrous nodule impinging against the prosthetic component in the intercondylar notch.

Ultrasound is particularly useful in this condition, since metal artifacts limit CT and MR imaging. Patellar clunk can be seen in the sagittal and axial plane as an echogenic lesion attached posteriorly to the quadriceps insertion onto the patella. ${ }^{50}$ The nodule pops out of the notch during extension when the transducer is placed over the patellofemoral compartment with the patient flexing and extending the knee. ${ }^{8}$

\section{Muscle herniation}

Muscle herniation is defined when a fascial defect allows herniation of a focal area of muscle. ${ }^{51}$ These typically occur in the lower leg, specifically the tibialis anterior muscle. Less commonly, the upper extremity is involved. Causes include sporting activities, prior trauma, chronic compartment syndrome, and weak overlying fascia due to perforating vessels..$^{52}$ The muscle protrudes and clinically presents most often as a painless soft-tissue mass, which appears as a focal swelling. Increased conspicuity is reported after exercise, and the condition is common in teenagers and young adults. ${ }^{52}$ Occasionally, there is severe pain or cramps and surgical intervention is required.

On ultrasound, fascia are typically thin and echogenic. Thinning and slight elevation of the fascia can suggest herniation. With dynamic ultrasound, the herniation appears as focal herniating muscle that is more prominent with 
muscle contraction. The herniated muscle may be hypoechoic due to anisotropy or atrophy. A mushroom-like appearance occurs when it overlaps the fascial defect and has a convex superficial contour (Figure 7). ${ }^{52,53}$ Additionally, pressure applied to the herniated muscle with the probe can often reduce the muscle. Alternatively, muscle contraction or standing position may reduce the muscle herniation through the fascial defect.

\section{Ankle and foot Syndesmosis injury}

High ankle sprains are uncommon, representing 1\%-11\% of all ankle injuries. ${ }^{54}$ The anterior inferior tibiofibular ligament (AITFL) is a flat, strong ligament that holds the fibula tight to the tibia and prevents excessive fibular movement and external talar rotation. It originates at the anterior aspect of the lateral malleolus and attaches on the anterolateral tubercle of the tibia.

Patients with syndesmotic ankle injury may have negative radiographs. Dynamic ultrasound has recently been shown to be highly specific and sensitive for AITFL injury compared to MRI. ${ }^{55}$ Normal anterior clear space, being the anterolateral aspect of the ankle at the level of the distal tibiofibular syndesmosis, in neutral position is $3.78 \mathrm{~mm}$ with a mean widening of $0.3 \mathrm{~mm}$ for external rotation to neutral and $0.44 \mathrm{~mm}$ for external rotation to internal rotation of the foot. ${ }^{56}$ Widening of $>5.06 \mathrm{~mm}$ is considered abnormal and a sign of AITFL tear, in addition to hypoechoic areas or discontinuity of the ligament on ultrasound. ${ }^{56}$

\section{Tibialis posterior tendon dislocation}

The tibialis posterior tendon can dislocate due to prior trauma, though this is a rare occurrence. This typically occurs when the foot is inverted and either dorsiflexed or plantarflexed with a violent contraction of the tendon. Patients typically experience prolonged medial ankle symptoms. The posterior tibial tendon courses behind the medial malleolus, and is held in the retromalleolar groove by the flexor retinaculum and fibroosseous tunnel. ${ }^{57}$ Tears of the retinaculum and fibro-osseous tunnels allow the tendon to dislocate anteriorly over the medial malleolus. Under dynamic ultrasound, with the transducer at the level of the medial malleolus in the axial plane, dorsiflexion of the foot will demonstrate abnormal anterior shifting of the posterior tibial tendon. ${ }^{8,57}$

\section{Peroneal tendon subluxation}

The peroneal tendons are primarily evertors of the foot and dynamic stabilizers of the lateral ankle. They also assist in ankle plantar flexion and pronation and abduction of the foot. ${ }^{58}$ Peroneal tendon subluxation is an uncommon entity, seen in $0.3 \%-0.5 \%$ of patients. Peroneal tendon dislocation is often secondary to prior trauma and tear of the superior peroneal retinaculum. It has been reported in acute athletic injuries, including skiing, ice-skating, running, soccer, and American football. Patients report a snapping or popping at the time of injury with swelling, redness, and pain at the fibular tip. Chronic subluxation is seen in patients with snapping or popping and lateral ankle pain, often due to chronic inversion ankle sprains, leading to repeated injury.

Dynamic ultrasound has been shown to have a $100 \%$ positive predictive value for demonstrating peroneal subluxation. ${ }^{59}$ On dynamic ultrasound imaging, the peroneal tendons may be seen dislocating anterolaterally over the lateral malleolus when a patient dorsiflexes and everts the ankle. The transducer is held in the transverse plane at the level of the lateral malleolus. In some cases, the superior peroneal retinaculum is intact, but there is still abnormal subluxation. In these patients, with dynamic dorsiflexion and eversion of the foot, the peroneal longus will be seen moving deeply and anteriorly relative to the

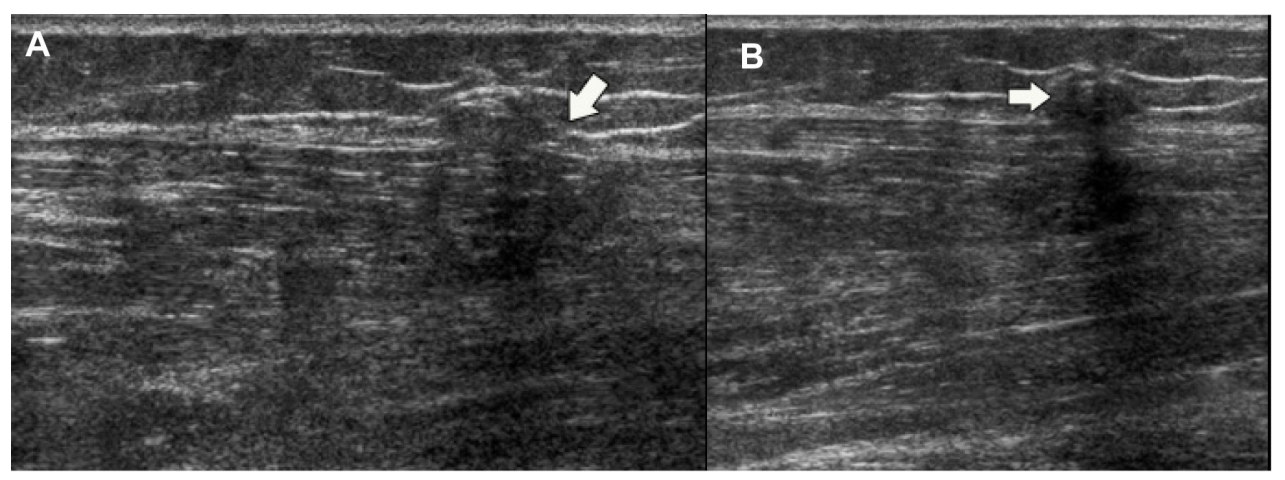

Figure 7 (A and B) Muscle herniation. (A) Prestanding longitudinal ultrasound image of the anterior tibialis muscle shows a focal defect (arrow). (B) Poststanding longitudinal ultrasound image shows focal herniation of hypoechoic muscle through the defect, with a mushroom-like appearance (arrow). 
brevis in the peroneal groove. ${ }^{59}$ Normally, the brevis is anterior to the longus.

\section{Orthopedic hardware}

Impingement of orthopedic hardware on soft tissues can result in local pain, derangement, and functional disability. Evaluation of impingement may be difficult with MRI and CT due to metal artifacts. No metal artifact is present with sonography. Additionally, dynamic imaging is particularly useful in this impingement syndrome to perform the motion that elicits patient symptoms. On ultrasound, metal hardware is hyperechoic with posterior ring-down artifact due to its strong specular reflective surface. ${ }^{60}$ Diagnosis of hardwareimpingement syndrome occurs when there is pathological change in structures adjacent to the hardware, and the patient has tenderness on the dynamic examination (Figure 8). Pathological changes include swollen, hypoechoic tendon sheath, tenosynovitis of the sheath, hypoechoic tendon with internal vascular changes, bursitis, and partial or complete tendon tears. ${ }^{60}$

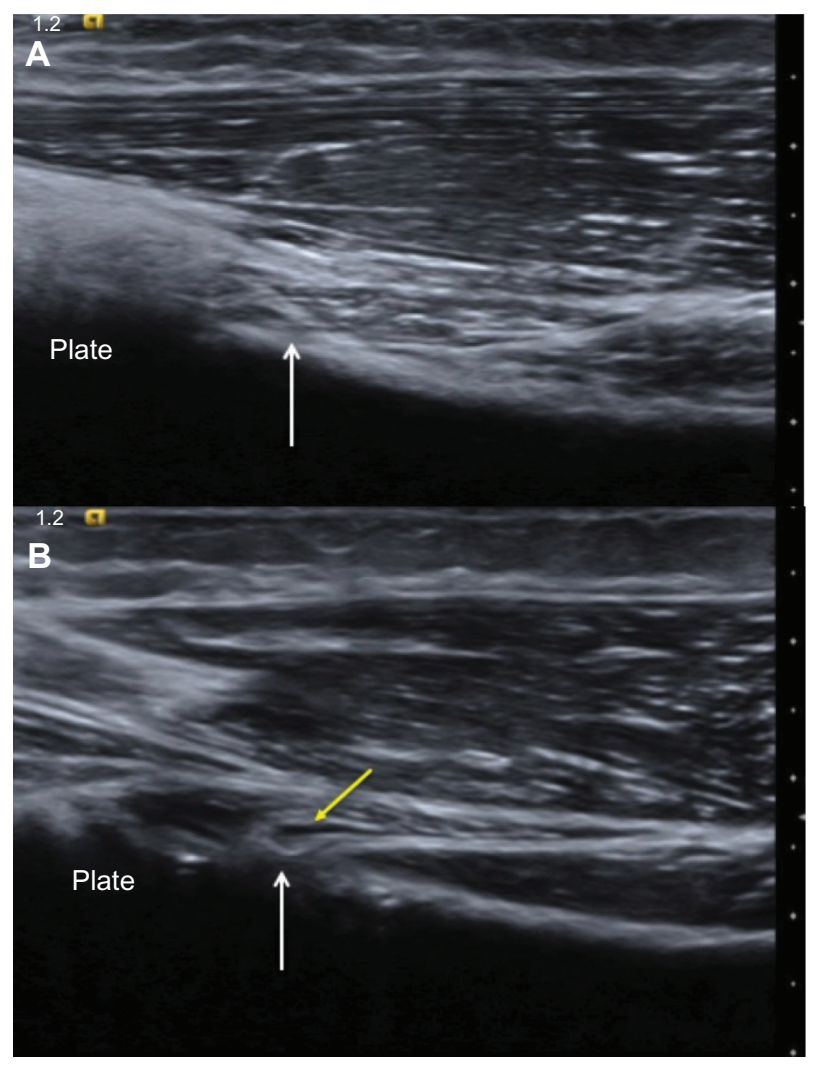

Figure 8 (A and B) Hardware impingement. (A) Longitudinal ultrasound image of the biceps (arrow) attachment onto the radial tuberosity shows normal attachment, without impingement from more distal shadowing plate. (B) Longitudinal ultrasound image during dynamic pronation of the forearm shows kinking of the biceps tendon (white arrow) due to the adjacent plate and small amount of peritendinous fluid (yellow arrow) due to the hardware impingement on the tendon.

\section{Recent research \\ Multivariate analysis of congruent images and orthogonal projections to latent structures}

New innovations in dynamic ultrasound imaging include the use of statistical analysis with computer mapping to create standards of normal skeletal muscle movement. Most measurements of tendon motion require tracking an area of small motion within the imaging window using B-mode ultrasound. ${ }^{61}$ Several studies have recently reported using multivariate analysis of congruent images (MAC) combined with partial least-squares regression and orthogonal projections to latent structures (O2PLS). MAC is utilized to compare ultrasound image sequences via discrete wavelet transformation. The wavelet extracts the position size and shape of the information present in a grayscale B-scan ultrasound image during muscle movement. ${ }^{1}$ O2PLS creates a model linking the two matrices, which in dynamic ultrasound could represent two ultrasound sequences of two movements by either the same subject or by different subjects. ${ }^{62}$ The difference in a region of interest on the image is tracked by a computer between the different sequences. ${ }^{1}$

Initial reports of Achilles tendon movement and biceps brachii movement using these techniques have been successful for observation of identical dynamic behavior in both sequences. Thus, the authors believe by analyzing two fulllength registrations simultaneously, sequences from healthy and injured patients could be compared or even in the same patient before and after intervention. ${ }^{1}$

\section{Elastography}

Another area of avid research involves the MSK applications of elastography. Elastography is clinically reported for evaluation of breast lesions and pathology in the liver. The technique is based on the principle of tissue deformability with pressure application, which is a dynamic maneuver. The information is represented on the monitor as blue in hard areas, red in soft areas, and green in firm areas with intermediate consistency. ${ }^{63}$ For MSK applications, an 8-12 MHz elastography-compatible linear probe in B-mode ultrasound on the right and color-coded sonoelastogram on the left are used.

In the Achilles tendon, tendinopathy appears as red rather than the normal blue, even before changes are seen on B-mode ultrasound. ${ }^{63}$ It has also been used to detect fatty atrophy, comparable to that in MRI, of the supraspinatus tendon in tears. ${ }^{64} \mathrm{~A}$ preliminary look at the use of elastography for the synovium has shown the ability to differentiate inflammatory 
from infectious synovitis. It can also differentiate malignant from benign synovial masses. In the synovium, malignant sarcomas show blue areas, while pigmented villonodular synovitis shows red to green with a few blue areas. On elastography, muscle is normally displayed as green. In advanced stages of inflammatory myositis, the muscle appears blue due to fibrosis. Inflamed nerves also have elastography changes, appearing red secondary to edema..$^{63,64}$

\section{Muscle quantification}

Determination of skeletal muscle cross-sectional area (CSA) and volume is useful in management of patients with sarcopenia, cachexia, and limb-muscle atrophy. ${ }^{65} \mathrm{MRI}$ and CT have been the gold-standard modalities for measuring CSA and volume. New research by Scott et al showed high agreement between MRI and panoramic ultrasound technique for measuring rectus femoris, gastrocnemius, and vastus lateralis muscle CSA and volume. ${ }^{65}$ Previous reports have shown promise for panoramic ultrasound in evaluation of the quadriceps muscle CSA. ${ }^{66,67}$ The ultrasound technique consists of B-mode axial plane images obtained with a $9 \mathrm{MHz}$ linear transducer in panoramic mode. Customized templates are placed on the thigh, and the sonographer takes a continuous, single view of the muscle by moving the probe across the thigh. The image is postprocessed into a two-dimensional cross-sectional image of the muscle. ${ }^{65}$

\section{Tissue-velocity imaging}

Tissue-velocity imaging (TVI) is a technique using modified color Doppler processing to image and quantify tissue motion. ${ }^{68}$ It has been used to quantify anatomy, regional movement, and myocardial velocity in the field of cardiology ${ }^{69}$ Several studies have reported using TVI during dynamic motion of skeletal muscle. ${ }^{68,70,71}$ It has potential use for resolving periodicity and timing of muscle motion during contractions, such as in fasciculation, tremors, and other neuromuscular disorders ${ }^{67}$ A recent study showed that using standard pulse-repetition frequency in TVI is accurate for skeletal muscle-tissue velocities above $0.17 \mathrm{~cm} / \mathrm{second}$, but must be adjusted for isometric muscle contraction or lowforce muscle contractions. ${ }^{67}$

\section{Speckle-tracking imaging}

Speckles are natural acoustic markers determined by interference patterns of tissue within an ultrasound window, allowing two-dimensional and three-dimensional sequences of tissue motion and deformation. ${ }^{72}$ A recent study by Peolsson et al showed isometric neck-muscle contraction visualized with ultrasound and tracked with speckle algorithm allowed for calculation of muscle-deformation rate and changes in muscle dynamics. ${ }^{73}$ Potential applications include understanding normal muscle-deformation rates to identify muscle abnormalities and target subsequent exercises and monitor rehabilitation progress. ${ }^{73}$

\section{Conclusion}

In conclusion, dynamic ultrasound imaging of the MSK system will likely become more frequent as health care reform pushes for less costly examinations, as the modality becomes more widely available, and referring physicians understand the benefit of dynamic imaging compared to MRI. The majority of pathology involves snapping of tendons along soft tissue or osseous structures. However, friction syndromes, impingement, and laxity are also potential applications. Future work is exciting, with promise in more tracking of patients using computer and statistical technology and comparing different patients. Additionally, sonoelastography is another potential open field for dynamic MSK ultrasound application.

\section{Disclosure}

The author reports no conflicts of interest in this work.

\section{References}

1. Löfstedt T, Ahnlund O, Peolsson M, Trygg J. Dynamic ultrasound imaging - a multivariate approach for the analysis and comparison of time-dependent musculoskeletal movements. BMC Med Imaging. 2012;12:29.

2. Sharpe RE, Nazarian LN, Parker L, Rao VM, Levin DC. Dramatically increased musculoskeletal ultrasound utilization from 2000 to 2009 , especially by podiatrists in private offices. $J$ Am Coll Radiol. 2012;9: 141-146.

3. Yablon CM, Wu JS, Newman LR, Downie BK, Hochman MG, Eisenberg RL. A needs assessment of musculoskeletal fellowship training: a survey of practicing musculoskeletal radiologists. AJR Am J Roentgenol. 2013;200:732-740.

4. Guillin R, Marchand AJ, Roux A, Niederberger E, Duvauferrier R. Imaging of snapping phenomena. Br J Radiol. 2012;85:1343-1353.

5. Neer CS 2nd. Anterior acromioplasty for the chronic impingement syndrome in the shoulder: a preliminary report. J Bone Joint Surg Am. 1972;54:41-50.

6. Fongemie AE, Buss DD, Rolnick SJ. Management of shoulder impingement syndrome and rotator cuff tears. Am Fam Physician. 1998;57: 667-674, 680-682.

7. Read JW, Perko M. Shoulder ultrasound: diagnostic accuracy for impingement syndrome, rotator cuff tear, and biceps tendon pathology. J Shoulder Elbow Surg. 1998;7:264-271.

8. Khoury V, Cardinal E, Bureau NJ. Musculoskeletal sonography: a dynamic tool for usual and unusual disorders. AJR Am J Roentgenol. 2007;188:W63-W73.

9. Bureau NJ, Beauchamp M, Cardinal E, Brassard P. Dynamic sonography evaluation of shoulder impingement syndrome. AJR Am J Roentgenol. 2006;187:216-220.

10. Roy A. Adhesive capsulitis in physical medicine and rehabilitation. 2012. Available from: http://emedicine.medscape.com/article/326828overview. Accessed September 20, 2013. 
11. Ryu KN, Lee SW, Rhee YG, Lim JH. Adhesive capsulitis of the shoulder joint: usefulness of dynamic sonography. $J$ Ultrasound Med. 1993;12:445-449.

12. Kim I, Yi JH, Lee J, et al. Limited subacromial gliding of the supraspinatus tendon during dynamic ultrasonography can predict a decrease in capacity and MR arthrographic features of the shoulder joint. Eur Radiol. 2012;22:2365-2670.

13. Bianchi S, Martinoli C. Ultrasound of the Musculoskeletal System. 1st ed. Berlin: Springer; 2007.

14. Armstrong A, Teefey SA, Wu T, et al. The efficacy of ultrasound in the diagnosis of long head of the biceps tendon pathology. J Shoulder Elbow Surg. 2006;15:7-11.

15. Teefey SA, Hassan SA, Middleton WD, Patel M, Wright RW, Yamaguchi K. Ultrasonography of the rotator cuff: a comparison of ultrasonographic and arthroscopic findings in one hundred consecutive cases. J Bone Joint Surg Am. 2000;82:498-504.

16. Farin PU, Jaroma H, Harju A, Soimakalio S. Medial displacement of the biceps brachii tendon: evaluation with dynamic sonography during maximal external shoulder rotation. Radiology. 1995;195:845-848.

17. Lazar MA, Kwon YW, Rokito AS. Snapping scapula syndrome. J Bone Joint Surg Am. 2009;91:2251-2262.

18. de Haart M, van der Linden ES, de Vet HC, Arens H, Snoep G. The value of computed tomography in the diagnosis of grating scapula. Skeletal Radiol. 1994;23:357-359.

19. Wood N, Konin JG, Nofsinger C. Diagnosis of an ulnar collateral ligament tear using musculoskeletal ultrasound in a collegiate baseball pitcher: a case report. NAm J Sports Phys Ther. 2012;5:227-233.

20. Nazarian LN, McShane JM, Ciccotti MG, et al. Dynamic US of the anterior band of the ulnar collateral ligament of the elbow in asymptomatic major league baseball players. Radiology. 2003;227: 149-154.

21. De Smet AA, Winter TC, Best TM, Bernhardt DT. Dynamic sonography with valgus stress to assess elbow ulnar collateral ligament injury in baseball players. Skeletal Radiol. 2002;31:671-676.

22. Konin GP, Nazarian LN, Walz DM. US of the elbow: indications, technique, normal anatomy, and pathologic conditions. Radiographics. 2013;33:E125-E147.

23. Spinner RJ, Goldner RD, Fada RA, Sotereanos DG. Snapping of the triceps tendon over the lateral epicondyle. J Hand Surg Am. 1999;24:381-385.

24. Spinner RJ, Goldner RD. Snapping of the medial head of the triceps: diagnosis and treatment. Tech Hand Up Extrem Surg. 2002;6:91-97.

25. Vanhees MKD, Geurts G, van Riet RP. Snapping triceps syndrome: a review of the literature. Shoulder Elbow. 2009;2:30-33.

26. Gupta VB, Patankar B, Paranjpe, Patil J. Ultrasound diagnosis of ulnar nerve dislocation and snapping triceps syndrome. SAfr J Radiol. 2012;16:65-67.

27. MacLennan AJ, Nemechek NM, Waitayawinyu T, Trumble TE. Diagnosis and anatomic reconstruction of extensor carpi ulnaris subluxation. J Hand Surg Am. 2008;33:59-64.

28. Allende C, Le Viet D. Extensor carpi ulnaris problems at the wrist classification, surgical treatment and results. J Hand Surg Br. 2005;30: 265-272.

29. Pratt RK, Hoy GA, Bass Franzcr C. Extensor carpi ulnaris subluxation or dislocation? Ultrasound measurement of tendon excursion and normal values. Hand Surg. 2004;9:137-143.

30. Lee KS, Ablove RH, Singh S, De Smet AA, Haaland B, Fine JP. Ultrasound imaging of normal displacement of the extensor carpi ulnaris tendon within the ulnar groove in 12 forearm-wrist positions. AJR Am J Roentgenol. 2009;193:651-655.

31. Fam AG. Regional pain problems. In: Klippel JH, Dieppe PA, editors. Practical Rheumatology. London: Mosby; 1997.

32. Kale S. Trigger finger. 2012. Available from: http://emedicine.medscape. com/article/1244693-overview. Accessed September 22, 2013.

33. Sato J, Ishii Y, Noguchi H, Takeda M. Sonographic appearance of the flexor tendon, volar plate, and A1 pulley with respect to the severity of trigger finger. J Hand Surg Am. 2012;37:2012-2020.
34. Serafini G, Derchi LE, Quadri P, et al. High resolution sonography of the flexor tendons in trigger fingers. J Ultrasound Med. 1996;15: 213-219.

35. Kim HR, Lee SH. Ultrasonographic assessment of clinically diagnosed trigger fingers. Rheumatol Int. 2010;30:1455-1458

36. Hame SL, Malone CP Jr. Boxer's knuckle: traumatic disruption of the extensor hood. Hand Clin. 2000;16:375-380.

37. Lopez-Ben R, Lee DH, Nicolodi DJ. Boxer knuckle (injury of the extensor hood with extensor tendon subluxation): diagnosis with dynamic US - report of three cases. Radiology. 2003;228:642-646.

38. Walker EA, Petscavage JM, Brian PL, Logie CI, Montini KM, Murphey MD. Imaging features of superficial and deep fibromatoses in the adult population. Sarcoma. 2012;2012:215810.

39. Schaberg JE, Harper MC, Allen WC. The snapping hip syndrome. Am J Sports Med. 1984;12:361-365.

40. Allen WC, Cope R. Coxa saltans: the snapping hip revisited. $J$ Am Acad Orthop Surg. 1995;3:303-308.

41. Cardinal E, Buckwalter KA, Capello WN, Duval N. US of the snapping iliopsoas tendon. Radiology. 1996;198:521-522.

42. Bae DK, Kwon OS. Snapping knee caused by the gracilis and semitendinosus tendon: a case report. Bull Hosp Jt Dis. 1997;56:177-179.

43. Marchand AJ, Proisy M, Ropars M, Cohen M, Duvauferrier R, Guillin R. Snapping knee: imaging findings with an emphasis on dynamic sonography. AJR Am J Roentgenol. 2012;199:142-150.

44. Lokiec F, Velkes S, Schindler A, Pritsch M. The snapping biceps femoris syndrome. Clin Orthop. 1992;283:205-206.

45. McAllister DR, Parker RD. Bilateral subluxating popliteus tendons: a case report. Am J Sports Med. 1999;27:376-379.

46. Fredericson M, Wolf C. Iliotibial band syndrome in runners: innovations in treatment. Sports Med. 2005;35:451-459.

47. Naredo E, Cabero F, Palop MJ, Collado P, Cruz A, Crespo M. Ultrasonographic findings in knee osteoarthritis: a comparative study with clinical and radiographic assessment. Osteoarthritis Cartilage. 2005;13:568-574.

48. Ko CH, Chang KK, Peng HL. Sonographic imaging of meniscal subluxation in patients with radiographic knee osteoarthritis. J Formos Med Assoc. 2007;106:700-707.

49. Acebes C, Romero FI, Contreras MA, Mahillo I, Herrero-Beaumont G. Dynamic ultrasound assessment of medial meniscal subluxation in knee osteoarthritis. Rheumatology (Oxford). 2013;52:1443-1447.

50. Okamoto T, Futani H, Atsui K, Fukunishi S, Koezuka A, Maruo S. Sonographic appearance of fibrous nodules in patellar clunk syndrome: a case report. J Orthop Sci. 2002;7:590-593.

51. Beggs I. Sonography of muscle hernias. AJR Am J Roentgenol. 2003;180:395-399.

52. van Holsbeeck MT, Introcaso JH. Musculoskeletal Ultrasound. 2nd ed. St Louis: Mosby; 2001.

53. Jarrett DY, Kramer DE, Callahan MJ, Kleinman PK. US diagnosis of pediatric muscle hernias of the lower extremities. Pediatric Radiol. 2013;43 Suppl 1:S2-S7.

54. Norkus SA, Floyd RT. The anatomy and mechanisms of syndesmotic ankle sprains. J Athlet Train. 2001;36:68-73.

55. Milz P, Milz S, Steinborn M, Mittlmeier T, Putz R, Reiser M. Lateral ankle ligaments and tibiofibular syndesmosis. 13-MHz high-frequency sonography and MRI compared in 20 patients. Acta Orthop Scand. 1998;69:51-55.

56. Mei-Dan O, Kots E, Barchilon V, Massarwe S, Nyska M, Mann G. A dynamic ultrasound examination for the diagnosis of ankle syndesmotic injury in professional athletes: a preliminary study. Am J Sports Med. 2009;37:1009-1016.

57. Goucher NR. Dislocation of the posterior tibial tendon: a literature review and presentation of two cases. Iowa Orthop J. 2006;26:122-126.

58. Clarke HD, Kitaoka HB, Ehman RL. Peroneal tendon injuries. Foot Ankle Int. 1998;19:280-288.

59. Neustadter J, Raikin SM, Nazarian LN. Dynamic sonographic evaluation of peroneal tendon subluxation. AJR Am J Roentgenol. 2004;183:985-988. 
60. Guillin R, Bianchi S. Sonographic assessment of orthopedic hardware impingement on soft tissues of the limbs. J Ultrasound. 2012;15:50-55.

61. Peolsson M, Löfstedt T, Vogt S, Stenlund H, Arndt A, Trygg J. Modelling human musculoskeletal functional movements using ultrasound imaging. BMC Med Imaging. 2010;10:9.

62. Trygg J, Wold S. Orthogonal projections to latent structures (O-PLS). J Chemom. 2002;16:119-128.

63. Lalitha P, Reddy MB, Reddy KJ. Musculoskeletal applications of elastography: a pictorial essay of our initial experience. Korean $J$ Radiol. 2011;12:365-375.

64. Schreiber V, Smekal V, De Zordo T, Fink C, Feuchtner G, Klauser A. Real-time sonoelastography in rotator cuff imaging and comparison to magnetic resonance imaging as gold standard. 2009. Available from: http://rsna2009.rsna.org/search/event_display.cfm?em_id=8016421\& printmode $=$ Y\&autoprint $=$ N. Accessed November 27, 2013.

65. Scott JM, Martin DS, Ploutz-Synder R, et al. Reliability and validity of panoramic ultrasound for muscle quantification. Ultrasound Med Biol. 2012;38:1656-1661.

66. Ahtiainen JP, Hoffren M, Hulmi JJ, et al. Panoramic ultrasonography is a valid method to measure changes in skeletal muscle cross-sectional area. Eur J Appl Physiol. 2010;108:273-279.

67. Arbeille P, Kerbeci P, Capri A, Dannaud C, Trappe SW, Trappe TA. Quantification of muscle volume by echography: comparison with MRI data on subjects in long-term bed rest. Ultrasound Med Biol. 2009;35:1092-1097.
68. Grubb NR, Fleming A, Sutherland GR, Fox KA. Skeletal muscle contraction in healthy volunteers: assessment with Doppler tissue imaging. Radiology. 1995;194:837-842.

69. Lindberg F, Martensson M, Gronlund C, Brodin L. Evaluation of ultrasound tissue velocity imaging: phantom study of velocity estimation in skeletal muscle low-level contraction. BMC Med Imaging. 2013;13:16.

70. Lindberg F, Ohberg F, Granasen G, Brodin LA, Gronlund C. Pennation angle dependency in skeletal muscle tissue Doppler strain in dynamic contractions. Ultrasound Med Biol. 2011;37:1151-1160.

71. Peolsson A, Brodin LA, Peolsson M. A tissue velocity ultrasound imaging investigation of the dorsal neck muscles during resisted isometric extension. Man Ther. 2010;15(6):567-573.

72. Geyer H, Caracciolo G, Abe H, et al. Assessment of myocardial mechanics using speckle tracking echocardiography: fundamentals and clinical applications. J Am Soc Echocardiogr. 2010;23: 351-369.

73. Peolsson A, Löfstedt T, Trygg J, Peolsson M. Ultrasound imaging with speckle tracking of cervical muscle deformation and deformation rate: isometric muscle contraction of patients after anterior cervical decompression and fusion for cervical disc disease. Man Ther. 2012;17: $519-525$.
Reports in Medical Imaging

\section{Publish your work in this journal}

Reports in Medical Imaging is an international, peer-reviewed, open access journal publishing original research, reports, reviews and commentaries on all areas of medical imaging. The manuscript management system is completely online and includes a very quick and fair peer-review system, which is all easy to use.

\section{Dovepress}

Visit http://www.dovepress.com/testimonials.php to read real quotes from published authors. 